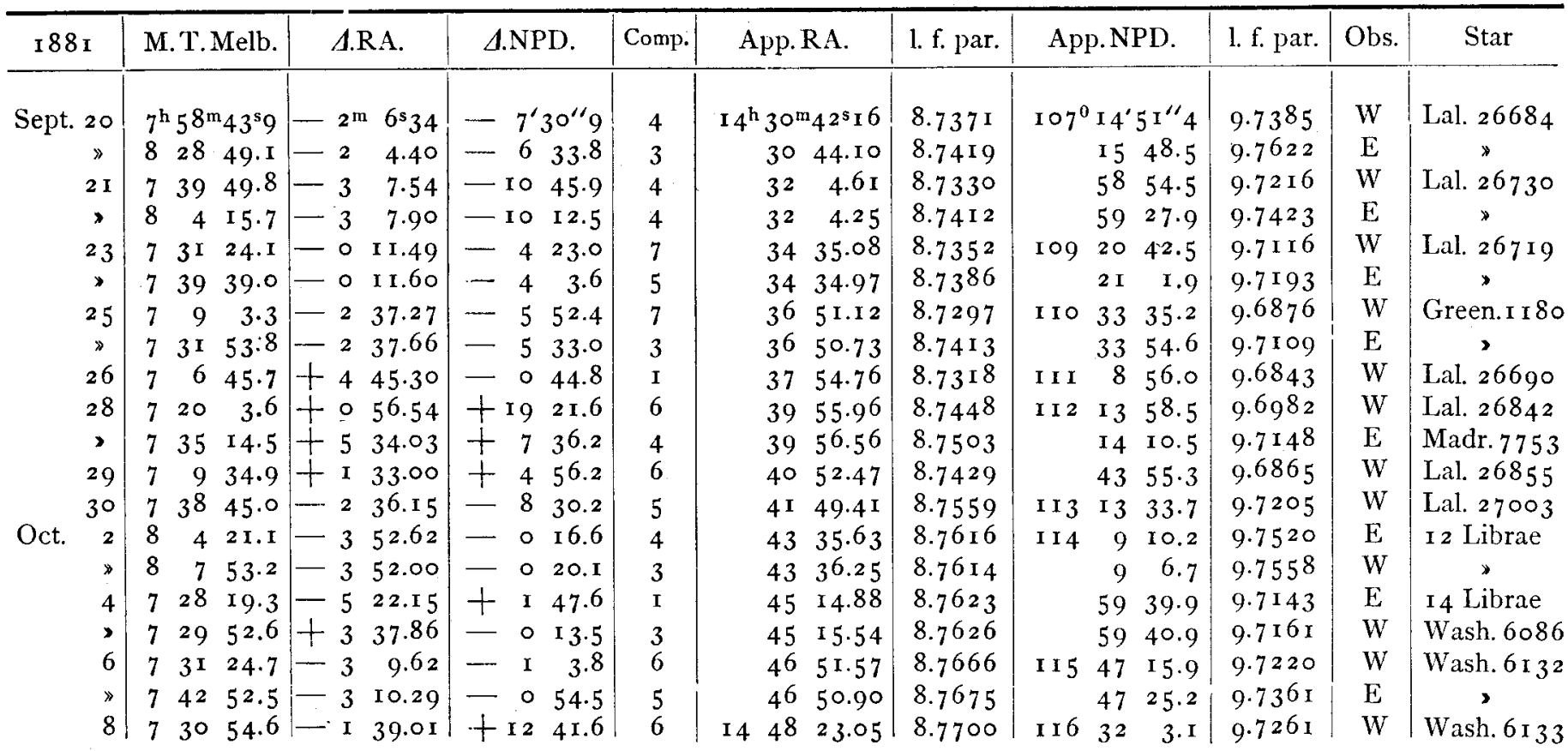

The observations marked $\mathrm{E}$ were made by Mr. Ellery $\mid$ faintly illuminated field. The places of Lalande's stars have with the South Equatorial of eight inches aperture, the micrometer had bright lines illuminated by means of a vacuum tube; those marked $\mathrm{W}$ were taken by Mr. White with the North Equatorial of four and a half inches aperture, the micrometer had wires thick enough to be seen with a

been taken from B. A. Lalande, $\mathrm{I}_{2}$ and $\mathrm{x}_{4}$ Librae from the Greenwich Catalogue for 1860 , and the others from the catalogues as named.

Melbourne I 88 I Oct. 26.

\title{
Beobachtung des Cometen Swift (entd. Nov. 16) und Ephemeride.
}

Von Herrn F. Palisa, Adjunct der K. K. Sternwarte in Wien.

Die letzte hier gelungene Beobachtung des Cometen Swift lautet:

Dec. 25 I $^{\mathrm{h}} 9^{\mathrm{m}} 5 \mathrm{I}^{\mathrm{s}}$ m. W.Z. $\alpha=23^{\mathrm{h}} 35^{\mathrm{m}} 4 \mathrm{o}^{\mathrm{s}} 29 \quad$ l. f. p. $9.63^{2}$ $\delta=+28^{0} 7^{\prime} 25^{\prime \prime} 5$ l. f. p. 0.700 Die Corr. meiner Ephemeride beträgt daher: $+o^{\circ} 5 \mathrm{u}$. $+\circ^{\prime} 6$

Nachdem zu erwarten ist, dass die Abweichung von der Ephemeride nicht so gross werden dürte, dass sie die Auffindung nach dem Vollmonde erschweren würde, so habe ich blos eine Fortsetzung der in No. 2408 mitgetheilten Ephemeride gerechnet.

Mittleres Aequ. I882.0.

\begin{tabular}{|c|c|c|c|c|}
\hline Mittl. Berl. Zeit & $\alpha$ & $\delta$ & $\log _{x} 4$ & $\mathrm{H}$. \\
\hline I 882 Jan. 6.5 & $23^{h} 3^{6 m} 59^{s}$ & $+20^{0} 3^{6} 5$ & $0.29^{6} 7$ & 0.33 \\
\hline $\begin{array}{l}7 \cdot 5 \\
8.5\end{array}$ & $\begin{array}{ll}37 & 16 \\
37 & 33\end{array}$ & $\begin{array}{lr}20 & 5.6 \\
\text { I } 9 & 37.6\end{array}$ & 0.3070 & \\
\hline $\begin{array}{r}9.5 \\
\text { Währir }\end{array}$ & 3752 & $\begin{array}{l}9 \quad 9.8\end{array}$ & & \\
\hline
\end{tabular}

\begin{tabular}{|c|c|c|c|c|}
\hline Mittl. Berl. Zeit & $\alpha$ & $\delta$ & $\log A$ & $\mathrm{H}$. \\
\hline I 882 Jan. I 0.5 & $23^{\mathrm{h}} 3^{8 \mathrm{~m}}$ I $2^{\mathrm{s}}$ & $+18^{\circ} 42^{\prime} 8$ & 0.3187 & \\
\hline I I. 5 & $3^{8} 3^{2}$ & I $8 \quad 16.4$ & & \\
\hline I 2.5 & 3853 & I $75 \mathrm{I} .5$ & $0.3^{2} 9^{2}$ & \\
\hline I 3.5 & 39 I 5 & I $7 \quad 27.4$ & & \\
\hline I 4.5 & 3938 & I $7 \quad 3.5$ & 0.3394 & \\
\hline I $5 \cdot 5$ & 402 & I 640.6 & & \\
\hline I 6.5 & 4027 & $16 \quad 18.4$ & 0.3494 & \\
\hline I 7.5 & 4053 & I $5 \quad 56.9$ & & \\
\hline I 8.5 & 4 I 19 & I 536.1 & 0.3590 & \\
\hline I 9.5 & $4 I \quad 45$ & I $5 \quad 15.9$ & & \\
\hline 20.5 & 42 I 2 & I 456.3 & 0.3684 & \\
\hline $2 \mathrm{r} .5$ & 4240 & I $437 \cdot 3$ & & \\
\hline 22.5 & 438 & I 48.9 & 0.3775 & \\
\hline 23.5 & $43 \quad 37$ & $14 \quad 1.0$ & & \\
\hline 24.5 & 447 & I 343.8 & 0.3862 & 0,20 \\
\hline
\end{tabular}

Die Helligkeit von Nov. 22 ist als Einheit angenommen. 7. Palisa.

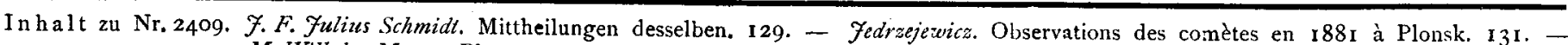
M. Wilhelm Meyer. Phaenomene der Jupiterstrabanten, I35. - B. v. Engelhardt. Beobachtungen des Cometen Swift (entd. Nov. I6). I 39. - B. A Gould. Beobachtungen des Cometen 188I IV auf der Sternwarte zu Cordoba. I39. - P. Tacchini. Osservazioni della cometa di Encke. I41. -- Robert $\mathcal{F}$. Ellery. Beobachtungen des Cometen I88I IV auf der Sternwarte zu Melbourne. I4I. - F. Palisa. Beobachtung des Cometen Swift (entd. Nov. I6) und Ephemeride. I43. 